\title{
Effect of Visualization on Muscular Activation for Stability in Adolescent Ballet Students
}

\author{
Emily Zhang ${ }^{1}$ and Jacqueline Katz ${ }^{\#}$ \\ ${ }^{1}$ Princeton High School, Princeton, NJ, USA \\ \#Advisor
}

$\underline{\text { ABSTRACT }}$

The purpose of this study is to determine whether commonly used visualization techniques, whose results have been solely anecdotal, produce tangible, scientific results in muscular activation and improvement to ballet balances. Ballet training methods include imagery techniques however, much of this practice is solely based on the experience of the instructor and its results are anecdotal so that there are many gaps between research on imagery and dance instruction. Few published studies focus on the effect of the imagery training for dance students on either motor and nonmotor outcomes (Abraham, 2019). A survey was administered to ballet instructors to determine the most used visualization cues for stability. Three adolescent female ballet students studying under said instructors were asked to perform three balances. Surface electromyography data was taken on the gluteus maximus, hip adductors, and abdominal oblique. The length of balance will also be taken. The dancers were then exposed to a short visualization session or stimulus of anatomical images with arrows showing bodily adjustments and targeted muscles accompanied by verbal cues developed based on the instructor techniques from the survey. The same balances and data was taken following the session. Results were compared to the control data taken prior to the session to reveal whether the visualization training had significant results by determining statistically significant changes in balance times and changes in neuron spikes following spike analysis. Dancers were also asked for qualitative feedback. Subject 2 yielded a significant increase in length of balance in all three types and the most consistent increase in neuron spikes in all of their muscles. This suggests a positive correlation between an increase in the degree of neuron activation or recruitment of those stability muscles and the ability for an individual to balance. This was also supported by increased confidence they felt in their balances after the visualization session. Subject 1 yielded no significant change in balance time before and after the visualization stimulus and the number of neuron spikes decreased after the session. This suggests that decreased activity in the tested muscles for stability resulted in lower balance times. This lack of muscular activation could be attributed to fatigue as reported by the dancer. The rest of the balances yielded significant increases in lengths of balance which were accompanied by increases in neuron spikes in the gluteus maximus and hip adductors for Degage a la Seconde and in the gluteus maximus for Releve en Retire. Subject 3 yielded insignificant changes in balance times for the first two types of balances but produced increases in the number of neuron spikes in most of the tested muscles in all of the balances. Reports from the dancer of being "less wobbly" the unexpected data to be attributed to an allocation to quality of the balance. The results on length of balances, number of neuron spikes, and confidence/reflection feedback obtained by this study supports the scientific validity of commonly-used visualization techniques in ballet by showcasing a higher degree of activation in the targeted stability muscles and longer average balance lengths should ensue following visualization training. Results also suggest that visualization techniques and stimuli for stability are the most effective when applied to learning unfamiliar movements. Further research could apply such visualization techniques to other movements, and even outside of dance. 


\section{Purpose}

Classical ballet is a unique area of study, notably for its extreme posture and resulting unique recruitment of muscles to maintain postural stability. To help students achieve greater stability, ballet training methods use visualization to activate muscles. Visualization or imagery in ballet can be defined as a mental experience that allows one to feel an appointed sensation representative of an actual experience. However, the results of visualization in ballet education are primarily anecdotal. The purpose of this study is to determine whether these commonly used visualization techniques produce tangible results in muscular activation in ballet balances so that anecdotal evidence can be scientifically backed.

\section{Literature Review}

Dance is unique in itself due to its demand for ideal physical attributes that are just as important as athleticism, performance, musicality, and technique skills (McCormack, 2018). Ballet is even more so. Stability is incredibly important as many steps in classical ballet require impressive feats of it, specifically dynamic stability defined as a type of postural stability and refers to the ability to remain upright during movement and requires strength, flexibility and proprioception (Paris-Alemany, 2018). In order to achieve this, correct activation of the muscles is necessary which stems from voluntary muscle contractions which are initiated when the central nervous system recruits motor units making up the muscles by activating their motor neurons (Stashuk, 2014). These connections can be better formed when the brain is given an image or visualization to guide the neural connection by focusing one on posture and anatomy based on biomechanics. Adherence to normal biomechanics allows for better functioning and injury prevention in dance.

A deviation from normal biomechanics often leads to injury in the foot and ankle (Ahonen, 2008). An example of the effect of unnatural movement is the demand for external hip rotation which challenges hip joint stability, and therefore requires the use of deep hip rotator muscles (Mayes, 2018). If these specific muscles that are not typically used in normal movement are not following normal biomechanics, there will be injuries. According to a study on injury patterns in professional ballerinas, $32 \%$ to $51 \%$ of the dancers were injured each year (Gamboa, 2008). This frequency of injury requires there to be a deeper understanding of the structure, biomechanics, and physics of the dancer's body; information essential to diagnose and evaluate the mechanics behind these injuries (Ahonen, 2008). To prevent such injuries and adhere to biomechanics, ballet training methods often include imagery techniques that are used to help improve dancers' abilities, posture, and technique. However, much of this practice is solely based on the experience of the instructor and its results are anecdotal (Abraham, 2019). This means that there are many gaps between research on imagery and dance instruction (Abraham, 2019). In fact, few published studies have actually been conducted with the focus on the effect of the imagery training for dance students on either motor and nonmotor outcomes (e.g. self-reported levels of improvement) (Abraham, 2019). Therefore, additional research on the effect of imagery on muscular activation would be greatly beneficial in adding to the pre-existing information that is very limited. This research could also benefit ballet training methods as it either determines a new method of imagery and visualization effective for dancers or confirms the effectiveness of previous practices. This study would majorly benefit dance research and consequently, dance training and injury prevention.

\section{Methods}

A survey was administered to ballet instructors to determine the most commonly used visualization methods for stability. Questions included those asking for the exact methods employed and what their intentions were. Three adolescent female ballet students studying under said instructors were asked to participate in a studio setting. These 
dancers were recruited for their similarity in age, level of training, and history of dance experience. Each were 16-17 years of age and have completed 10-14 years of ballet training.

Surface electromyography data was taken on the accessible gluteus maximus, hip adductor, and abdominal oblique muscles in three balancing steps: namely, releve in first position, degage a la seconde on a flat standing foot, and retire or passe en releve. The length of the balance was also recorded from the time at which the dancer's hand leaves the barre to the time at which it returns when they feel as if they are falling off balance. The dancers were then exposed to a visualization stimulus session using anatomical and structural images such as arrows depicting bodily adjustments and highlighting of targeted muscles accompanied by verbal cues.

This stimulus session was developed based on the commonly used techniques from the survey and was presented before each balance. The presentation of cues was short ( 2 minutes) and served as a stimulus for visualization techniques and its appointed sensations. The same data was taken following the session. Results were compared to the data taken prior to the session (the control) to reveal whether the visualization training had significant results by determining statistically significant changes in balance times before and after the stimulus, and changes in neuron spikes following spike analysis before and after the stimulus.

All participation is informed and consensual. Since minors are participating, parents or guardians are required to sign a release form. The ethics of this methodology have been reviewed and approved by an Institutional Review Board.

\section{Results}

By comparing the length of balance in seconds before and after the visualization stimulus within each test subject, the following was found. A spike analysis program of the raw EMG data signal detected the potential neuron spikes in the signal from spike candidates that represent a spike of the neuron we are interested in or the spikes of a distant neuron. These spikes data were found to have correlations to the increases in the length of balance before and after the visualization for each individual.

Subject 1: Balance Time \& Neuron Spike Results

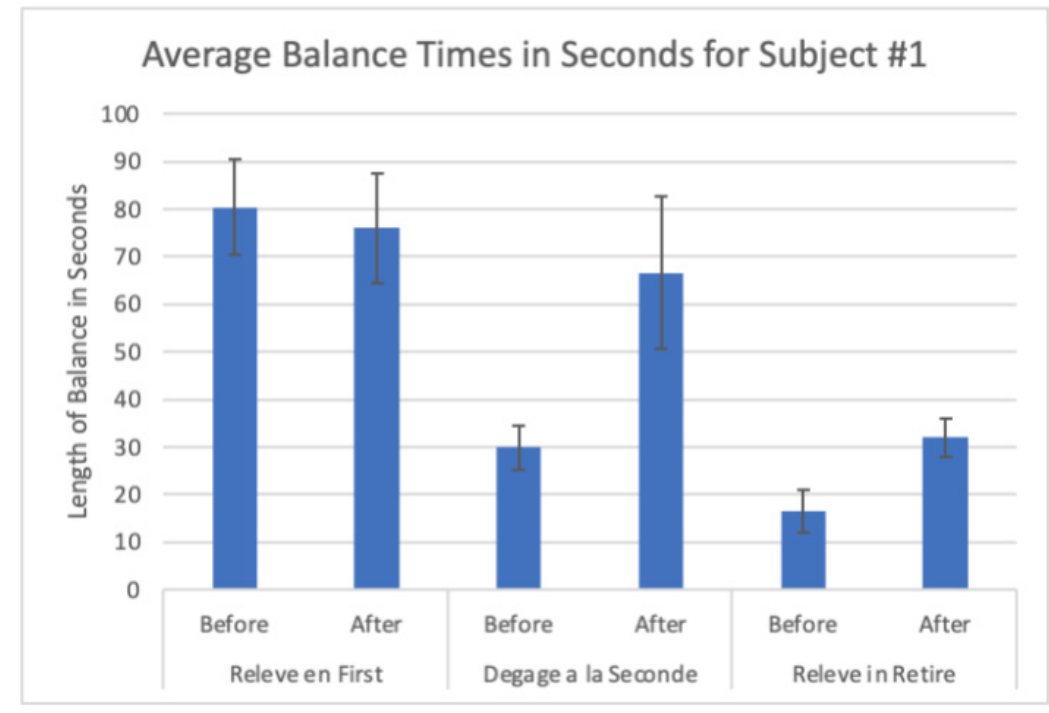

Figure 1. Average balance times in seconds for Subject 1. This bar graph depicts the average balance lengths of Subject 1 before and after a session of visualization training/stimulus. Releve en First shows a statistically significant change, however the rest of the balance show statistically significant results of an increase in balance time. 
Table 1. Number of Neuron Spikes for Each Muscle and Each Balance in Subject 1

\begin{tabular}{|l|l|l|l|}
\hline & & Number of Neuron Spikes \\
\hline Releve en First & & Before stimulus & After stimulus \\
\hline & gluteus maximus & 41414 & 9211 \\
\hline & inner thigh & 37885 & 7036 \\
\hline & core/oblique & 22810 & 6940 \\
\hline Degage a la Seconde & & & \\
\hline & gluteus maximus & 2716 & 3370 \\
\hline & inner thigh & 1936 & 4155 \\
\hline Releve en Retire & core/oblique & 7773 & 6097 \\
\hline & & & 4757 \\
\hline & gluteus maximus & 953 & 2817 \\
\hline & inner thigh & 2976 & 2613 \\
\hline & core/oblique & 5953 & \\
\hline
\end{tabular}

*Red represents a decrease, green represents an increase, and yellow represents an inconclusive change.

For Subject 1, there was no significant change before and after the visualization stimulus for Releve en First, however it was found that there was a significant increase in the length of balance in Degage a la Seconde (from $29.873 \pm 4.550$ seconds to $66.540 \pm 16.028$ seconds) as well as a significant increase in the length of balance in Releve en Retire (from $16.500 \pm 4.426$ seconds to $31.960 \pm 4.025$ seconds) (Figure 1).

For the balances in Subject 1 in which there was a significant increase in length of balance (Degage a la Seconde and Releve en Retire), there was a significant increase in the number of neuron spikes in some muscles before and after the visualization stimulus. For Releve en First, there was a decrease in the number of neuron spikes for each of the three muscles tested. For Degage a la Seconde, the number of neuron spikes increased from 2716 to 3370 spikes for the gluteus maximus muscle and from 1936 to 4155 spikes for the hip adductor muscles. There was not a significant increase in the number of neuron spikes for the oblique. For Releve en Retire, the number of spikes increased from 953 spikes to 2817 spikes in the gluteus maximus muscle. There was not a significant increase in the number of neuron spikes for the hip adductors or the abdominal oblique. 
Subject 2: Balance Time \& Neuron Spike Results

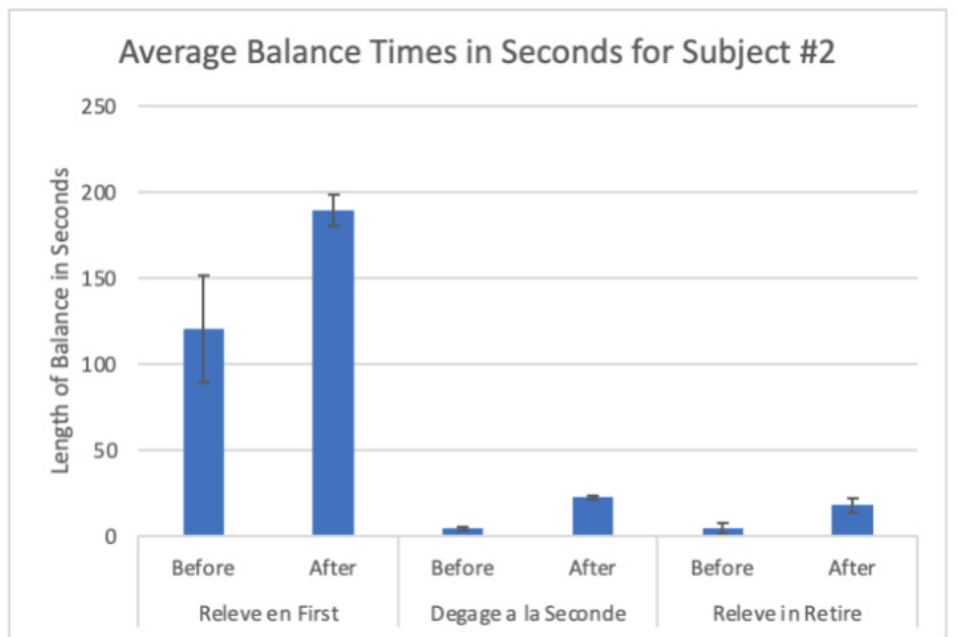

Figure 2. Average balance times in seconds for Subject 2. This bar graph depicts the average balance lengths of Subject 2 before and after a session of visualization training/stimulus. All of the tested balances show statistically significant results of an increase in balance time.

Table 2. Number of Neuron Spikes for Each Muscle and Each Balance in Subject 2

\begin{tabular}{|c|c|c|c|}
\hline \multirow[b]{2}{*}{ Releve en First } & & \multicolumn{2}{|c|}{ Number of Neuron Spikes } \\
\hline & & Before stimulus & After stimulus \\
\hline & gluteus maximus & $\mathrm{n} / \mathrm{a}$ & $\mathrm{n} / \mathrm{a}$ \\
\hline & inner thigh & $\mathrm{n} / \mathrm{a}$ & $\mathrm{n} / \mathrm{a}$ \\
\hline & core/oblique & 20224 & $\mathrm{n} / \mathrm{a}$ \\
\hline \multicolumn{4}{|c|}{ Degage a la Seconde } \\
\hline & gluteus maximus & 1140 & 2662 \\
\hline & inner thigh & 941 & 2173 \\
\hline & core/oblique & 1304 & 5696 \\
\hline \multicolumn{4}{|l|}{ Releve en Retire } \\
\hline & gluteus maximus & 1018 & 3456 \\
\hline
\end{tabular}




\begin{tabular}{|l|l|l|l|}
\hline & inner thigh & 2506 & 3276 \\
\hline & core/oblique & 3482 & 4578 \\
\hline
\end{tabular}

*Red represents a decrease, green represents an increase, and yellow represents an inconclusive change.

For Subject 2, there was statistically significant increase in length of balance before and after the visualization stimulus for all three of the balances. Releve in First yielded a change from $120.773 \pm 31.235$ seconds to $189.747 \pm$ 8.969 seconds, Degage a la Second yielded a change from $4.457 \pm 1.169$ seconds to $22.547 \pm 1.1425$ seconds, and Releve in Retire yielded a change from $4.807 \pm 2.804$ seconds to $17.9966667 \pm 3.830$ seconds (Figure 2).

For the balances in Subject 2 in which there was a significant increase in length of balance (all 3), there was a significant increase in the number of neuron spikes before and after the visualization stimulus in all of the muscles in Degage a la Second and Releve en Retire. The spikes for Releve en First were inconclusive. For Degage a la Seconde, the number of neuron spikes increased from 1140 to 2662 spikes for the gluteus maximus muscle, from 941 to 2173 spikes for the hip adductor muscles, and from 1304 spikes to 5696 spikes for the abdominal oblique. For Releve en Retire, the number of neuron spikes increased from 1018 to 3456 spikes for the gluteus maximus muscle, from 2506 to 3276 spikes for the hip adductor muscles, and from 3482 spikes to 4578 spikes for the abdominal oblique.

Subject 3: Balance Time \& Neuron Spike Results

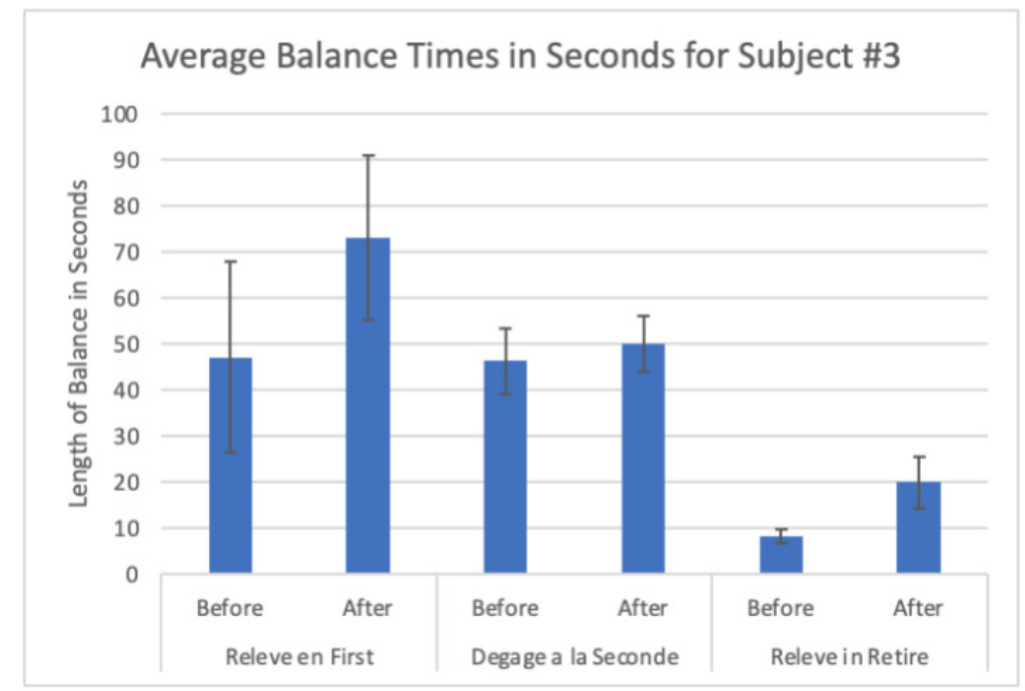

Figure 3. Average balance times in seconds for Subject 3. This bar graph depicts the average balance lengths of Subject 3 before and after a session of visualization training/stimulus. Releve en First and Degage a la Seconde show statistically insignificant change, however Releve Retire shows statistically significant results of an increase in balance time. 
Table 3. Number of Neuron Spikes for Each Muscle and Each Balance in Subject 3

\begin{tabular}{|c|c|c|c|}
\hline & & Number of Neurc & \\
\hline \multirow[t]{4}{*}{ Releve en First } & & Before stimulus & After stimulus \\
\hline & gluteus maximus & 5634 & 13665 \\
\hline & inner thigh & 27616 & $\mathrm{n} / \mathrm{a}$ \\
\hline & core/oblique & 13838 & 18975 \\
\hline \multicolumn{4}{|c|}{ Degage a la Seconde } \\
\hline & gluteus maximus & 2359 & 5733 \\
\hline & inner thigh & 4663 & 10361 \\
\hline & core/oblique & 11149 & 13313 \\
\hline \multicolumn{4}{|l|}{ Releve en Retire } \\
\hline & gluteus maximus & 1282 & 4717 \\
\hline & inner thigh & 1220 & 1254 \\
\hline & core/oblique & 4524 & 2069 \\
\hline
\end{tabular}

*Red represents a decrease, green represents an increase, and yellow represents an inconclusive change.

For Subject 3, there was no statistically significant significance change between the length of balance before and after the visualization stimulus in the first two balance types, however Releve en Retire yielded a significant increase in length of balance from $8.193 \pm 1.473$ seconds to $19.96 \pm 5.566$ seconds (Figure 3)

For the balances in Subject 3, there was a significant increase in some of the muscles for each of the balances before and after the visualization stimulus regardless of a significant increase in length of balance. For Releve en First, the number of neuron spikes increased from 5634 to 13665 spikes for the gluteus maximus muscle and from 13838 to 18975 spikes for the abdominal oblique. The change in the number of neuron spikes for the hip adductor muscle was inconclusive. For Degage a la Seconde, the number of neuron spikes increased from 2359 to 5733 spikes for the gluteus maximus muscle, from 4663 to 10361 spikes for the hip adductor muscles, and from 11149 spikes to 13313 spikes for the abdominal oblique. For Releve en Retire, the number of neuron spikes increased from 1282 to 4717 spikes for the gluteus maximus muscle and from 1220 to 1254 spikes for the hip adductor muscles. There was a decrease in the number of neuron spikes for the oblique. 


\section{Overall Qualitative Feedback}

Feedback from all of the dancers provided positive experiences following the visualization stimuli. Subject 1 used descriptors such as not getting "tired as fast" and feeling less "emphasis" or pressure in the "knees and the front of the hips" as effects of the visualization session. They remember "pulling up in the upper thigh" more and "using [their] core much more" after the session. On a scale of 1 to 5 where 1 is the least and 5 is the most, they responded with a 3 in regards to whether they felt as if they were activating different muscles more intensively following the visualization training session than before and a 4 in feeling more confident in balancing than before. Subject 2 provided descriptors such as thinking "more about the inner thighs" and the visualizations "kept [them] from falling off on the side" and that they "felt more grounded" and a sensation of "pulling in" that "kept the [standing] foot from turning in". They generally "felt more centered" following the visualization stimulus. On a scale of 1 to 5 where 1 is the least and 5 is the most, they responded with a 5 in regards to whether they felt as if they were activating different muscles more intensively following the visualization training session than before and a 4 in feeling more confident in balancing than before. Subject 3 used descriptors such as being able to "go up higher on releve" and a "wrapping" sensation of the "leg muscles" allowed their balance to be "more consistent and less wobbly" so that they felt "more stable as a whole". On a scale of 1 to 5 where 1 is the least and 5 is the most, they responded with a 5 in regards to whether they felt as if they were activating different muscles more intensely following the visualization training session than before and a 4 in feeling more confident in balancing than before.

\section{Discussion}

Visualization, imagery, and an individual's interpretation of it for its resulting muscle activation is unique and pertains to each individual differently. As a result, the obtained data varies from person to person. Notably, however, Subject 2 who most consistently had a significant increase in length of balance in all three types had the most consistent increase in neuron spikes in all of their muscles. This implies a clear positive correlation between an increase in the degree of neuron activation or recruitment of those stability muscles and the ability for an individual to balance. This change was especially observable in degage a la seconde in this subject which they could only hold for an exceptionally low amount of time prior to the visualization stimulus but increased significantly following the stimulus. This was supported by the consequent significant increase in neuron spikes in all of the stability muscles and the increased confidence they felt in their balances following the visualization session.

Subject 1 demonstrated correlation between the length of balance and change in number neuron spikes. There was no significant change in balance time before and after the training session and the corresponding neuron spike results provided that the number of neuron spikes actually decreased after the session as well. This implies that decreased activity in the tested muscles for stability results in lower balance times. This lack of muscular activation could be attributed to fatigue and possible confusion with these first visualization instructions as this dancer reported. The rest of the balances yielded significant increases in lengths of balance which were accompanied by increases in neuron spikes in the gluteus maximus and hip adductors for Degage a la Seconde and in the gluteus maximus for Releve en Retire. Differences in interpretation, individual biomechanics, and existing perception of the visualization cues provided all contribute to the differences in the muscles this dancer targeted as opposed to other dancers.

Subject 3 yielded interesting results as well considering their insignificant changes in balance times for the first two types of balances but still maintaining increases in the number of neuron spikes in most or all of the tested muscles in all of the balances. From the feedback provided by this dancer, the lack of increase in length of balance despite increases in neuron spikes can be attributed to a focus on quality of the balance. Reports from the dancer of being "less wobbly" and feeling the targeted sensations provided for by the visualization stimuli suggest this to be true. Though the increases found in the number of neuron spikes in the muscles only allowed for an increased length 
of balance for Releve en Retire and not the other two balances, the visualization stimuli provided a higher quality in posture and confidence of the balance for the dancer.

\section{Conclusion}

The overall comparison of results to determine change in length of balances, change in number of neuron spikes, as well as confidence and reflection feedback obtained by this study generally supports the scientific validity of commonly used visualization techniques in ballet by showcasing a higher degree of activation in the targeted stability muscles and longer average balance lengths should ensue following a session of visualization training/stimulus in most cases. The results obtained also support the claim that visualization allows a dancer to have a better understanding and clear reminder of the desired sensation of the muscles they are focused on activating allowing for more targeted muscle firing. The cases in which the change in length of balance was insignificant before and after the training session were primarily found to be in the balance types in which the dancers were already more familiar and confident with. The same applies to insignificant changes in stability muscles. This suggests that visualization techniques and stimuli for stability are the most effective when applied to learning new types of balances or more unfamiliar movements. This supports the effectiveness of the widespread use of visualization techniques in teaching, especially in the instruction on new movements. Further research could be conducted in this field by observing additional muscles, perhaps those that should be activated less, applying such visualization techniques to types of movement other than just balances, and even applying visualization outside of ballet to improve stability.

\section{Limitations}

This study was conducted throughout the Covid-19 pandemic safely which limited the sample size although this project tends more towards a case study so a large sample size is not necessary. Materials such as EMG technology was school-provided and future studies could obtain more accurate results through more advanced technology.

\section{Acknowledgments}

Thank you to the dancers and instructors of Princeton Dance \& Theater for their time and participation as well as Dr. Antonia Zaferiou \& Dr. Amit Abraham for their mentoring and consultation in this study.

\section{References}

Abraham, Amit, et al. "Dynamic Neuro-Cognitive Imagery (DNITM) Improves Developpé Performance, Kinematics, and Mental Imagery Ability in University-Level Dance Students.” Frontiers, Frontiers, 7 Feb. 2019, www.frontiersin.org/articles/10.3389/fpsyg.2019.00382/full.

Ahonen, Jarmo. "Biomechanics of the Foot in Dance." Journal of Dance Medicine \& Science, vol. 12, 2008, www.ncbi.nlm.nih.gov/pubmed/19618585.

Gamboa, Jennifer M., et al. "Injury Patterns in Elite Preprofessional Ballet Dancers and the Utility of Screening Programs to Identify Risk Characteristics.” Journal of Orthopaedic \& Sports Physical Therapy, vol. 38, no. 3, 2008, pp. 126-136., doi:10.2519/jospt.2008.2390.

Mayes, Susan, et al. “Obturator Externus Was Larger, While Obturator Internus Size Was Similar in Ballet Dancers Compared to Nondancing Athletes." Physical Therapy in Sport, vol. 33, 2018, pp. 1-6., doi:10.1016/j.ptsp.2018.06.001. 
McCormack, Moira, et al. "The Physical Attributes Most Required in Professional Ballet: A Delphi Study." Sports Medicine International Open, vol. 03, no. 01, 2018, doi:10.1055/a-0798-3570.

Paris-Alemany, Alba, et al. "Comparison of Lumbopelvic and Dynamic Stability between Dancers and NonDancers." Physical Therapy in Sport : Official Journal of the Association of Chartered Physiotherapists in Sports Medicine, U.S. 